\title{
Software system for solving multi-scale optimization problems
}

\author{
E. Semenkin, K. Abramovich \\ Siberian Aerospace Academy \\ P.O.Box 486, Krasnoyarsk, 660014, Russia. \\ Tel: +7-391-2-33-34-20. Fax: +7-391-2-33-47-09. \\ e-mail: semenkinestu.krasnoyarsk. su
}

\begin{abstract}
Optimization problems with different type of variables are described. Methods of decomposition and unification are suggested for solving these problems. Software system, that realizes mentioned methods, is described. Results of testing and ways of further development are discussed.
\end{abstract}

\section{Keywords}

Multi-scale optimization, decomposition, unification, software system

\section{EXAMPLES OF PROBLEM}

Optimization problems with the variables of different type (discrete, boolean, continuous, combinatorial, etc.) are called multi-scale or mixed problems. They arise in various design and planning problems when mathematical formulations and modelling are possible. Consider two examples of problems of that type. (subsystems).

1) $\mathrm{CAD}$ of some large scale system consisting of several interconnected units

The objective $F(B, D, X)$ is some quality index, representing the ability of the system to perform its functions. $B \in \mathbf{B}_{\mathbf{n}}$ is the vector of Boolean variables representing the system structure. If $b_{i}=1$ then $i$-th subsystem is included in the system and vice verse. $\mathbf{D} \in \mathbf{Z}_{m}$ is the vector of integer variables, representing the number of backup copies of subsystems. $\mathbf{X} \in \mathbf{R}_{\mathbf{K}}$ is the vector of continuous variables representing the physical characteristics of the subsystems (mass, power consumption, etc.).

2) Planning of optimal sequence of an industrial projects' implementation. 
Let some enterprise have several industrial investment projects. The problems are to choose the best ones, to define the sequence of their realization and to estimate the optimal values of project parameters. In this case, the objective $F(Y)=\left\{f_{1}(\mathbf{Y}), f_{2}(Y), \ldots, f_{k}(Y)\right\}$ is a multicriterial quality index, where, for example, $f_{1}(Y)$ - the value of necessary credit resources, $f_{2}(\mathbf{Y})$ - pay-back period, $f_{k}(\mathbf{Y})$ - maximal cash outflow, etc. $\mathbf{Y}=\{\mathbf{B}, \mathbf{C}, \mathbf{X}\}$ is the multi-scale vector of controllable variables. $\mathbf{B}$ is the vector of Boolean variables representing the totality of projects to be realized, $\quad C$ is the combinatorial variable defining the sequence of the projects, $\mathbf{X}$ is the vector of continuous variables describing some features of projects (production volume, cost structure, etc.).

Note that the quality index of such problems is usually multicriterial one and some of the criteria are given in implicit form, i.e., they have not evident analytical form but can be calculated algorithmically.

\section{METHODS OF DECOMPOSITION AND UNIFICATION}

Let's consider two basic approaches for solving the mixed optimization problems (Semenkin, 1992).

First approach, called decomposition method, has the following essence: the original multi-scale problem is replaced by several problems with variables of unique type. For this purpose the priority levels for each variable type are introduced, for instance: first (highest) level - Boolean variables; second level - discrete variables, etc. The multilevel optimization problem is then solved, a process, during which the solution of a lower-level problem corresponds to the evaluation of the objective function value in one current point for higherlevel problem. Optimization on each level is implemented by the algorithm of according type, independently on the other levels.

The advantages of this method are the following:

- it is possible to choose for each level the most suitable algorithm regarding the problem features;

- there is the possibility to introduce the levels' priority according to that of the real problem.

The disadvantage is the great laboriousness of the method, i.e., the necessity of the large number of objective function evaluations. The effectiveness of the method depends entirely on the effectiveness of involved algorithms.

Second approach for solving of mixed optimization problems is called method of variables' unification and has the following essence: variables of different type are replaced by the variables of unique type, namely by continuous (relaxation), integer (discretization) or by boolean (binarization) variables.

The replacing of all variables by continuous ones is the most suitable way from optimization point of view, because the mathematical programming methods are well-known and relatively powerful. However, as it was mentioned above, mixed optimization problems are described often by implicit objective function and, in this case, it is impossible to calculate the objective function value beyond the discrete lattice. That is why the relaxation was not used by the development of the software described in the next section.

Transformation of continuous variables into discrete (integer) ones is performed easily when an error margin of the minimum point location in continuous space is known and when 
left and right bounds are given. Boolean variables can be treated as an integer ones bounded by 0 and 1 . Thus one can use integer variables as the unification base.

In turn, discrete variables can be transformed to boolean ones using binary code of integers. Hence unification to boolean variables is possible too.

The advantage of the unification method is essentially lower number of objective function evaluations in comparison with decomposition method. However, there are some disadvantages that cannot be neglected.

The first of them is that the treatment of boolean variables in form of integers, bounded by 0 and 1 , with discrete optimization algorithms leads, in some cases, to the bad work of these algorithms, that are not destined to this situation.

Other disadvantages, related to the binarization method, are the following:

- difference of topological properties in original space and in binarized one (near points become far ones and vice versa), which leads to the loss of useful properties of the objective function (monotonicity, convexity, etc.),

- considerable growth of problem dimension in comparison with the dimension of an initial problem,

- existence of relatively large number of points in binarized space that have no corresponding points in initial space.

\section{OPTIMIZATION SUPPORT SYSTEM}

Approaches described above (decomposition and unification using integer and boolean variables) were realized in the software system for solving mixed optimization problems (Abramovich, 1995). Aim of this software is to supply the convenient tool and to guide the user by solving problems of such type. This software can be also used for solving usual (not mixed) optimization problems, both continuous, and discrete and boolean ones. The system was developed to be flexible in sense of modifications.

Optimization algorithms included in the system, are of 3 type: continuous, boolean and discrete. The origin of them is different. Some algorithms were taken from other optimization systems (Dolezal and Fidler, 1992). Rest of them was developed in Siberian Aerospace Academy (Semenkin and Semenkina (1994), Semenkina (1995)). All of these algorithms are direct search methods of different kinds. This method was chosen because of its universality and applicability to implicit objective functions. The algorithm library is not reach at present, but there is a possibility to add easily any necessary algorithm.

Besides the algorithm library there are the following elements of the software system:

- C compiler, which is aimed at the creation of an executable program code after the preparation of initial data,

- interface program which interacts with the user of the system and manages the process of work,

- interface files used for introduction of initial data and for interacting of optimization algorithms with the main program,

- objective function prototype that should be prepared by user according to the problem solved.

Let's consider the sequence of steps during the work with software system. 
The first step is the choice of the solving method (decomposition, discretization or binarization). If decomposition method was chosen, the user has to introduce priority levels for each type of variables presenting in the problem.

The second step is the choice of an optimization algorithm from the list of available ones. After that, some initial data should be introduced to the interface files.

The third step is the introduction of the objective function. This function can be of any complexity. The programming language is Microsoft $\mathrm{C}$, version 6.0.

The fourth step is the executable program generation and implementation. After that, the user can change either optimization algorithm or objective function or method of problem solving and repeat the described steps, or the work with the system can be finished.

On-line help is available during all the steps of a problem solving.

\section{4}

RESULTS OF TESTING AND FURTHER DEVELOPMENTS

After the software was developed some test problems were solved using this system. Objective functions with variables of different type were taken and treated by different methods and algorithms. The results were different within the same method, depending on the algorithms applied, but in all cases a local minimum point was found.

Testing of optimization methods allows to draw some useful conclusions:

- unification method operates essentially faster and needs less number of objective function evaluation in comparison to decomposition,

- direct treatment of boolean variables in form of integers, bounded by 0 and 1 , leads, in some cases, to the bad work of discrete algorithms. The most evident reason for that is the impossibility to define search direction in boolean space.

- binarization method leads to the steep increase of the problem dimension and hence to the very slow work of boolean optimization algorithm. To overcome this effect the adaptive approach for the choice of the search accuracy for continuous variables was realized, the essence of which is that this accuracy is not fixed but changes step by step to keep the number of points relatively small.

Some real problems of design of spacecraft's' systems were solved with developed software system. These problems are:

- Ioad planning for subsystems of the control complex of the orbital group of communication satellites (Abramovich, 1995),

- maximization of expected profit when exploitation of communication satellites (Semenkina, 1995),

- optimal choice of the variant of technological contour of the control system of orbital group of spacecrafts (Semenkina, 1995).

When solving mentioned real problems, developed software system has showed good workability and allowed to obtain the results, that were interesting for developers of space systems.

There are several directions of the software system development.

The first (and the most evident) direction is the addition of new optimization algorithms to the library. It is necessary to add searching algorithms with global properties (multistart of local search) and to introduce one more variable type (combinatorial one) after development of 
according algorithms. It seems reasonable also to include such promising and powerful algorithms of adaptive random search as Genetic and Simulating Annealing ones, which are currently under development. The last direction of the expansion of algorithm library is the development of the algorithms that realize main approaches for multicriterial decision making.

Other direction of the system improving is connected with the realization of newly appeared ideas about using decomposition and unification methods.

The first idea is to apply the decomposition approach not only for variables of different type but for variables of the same type. For example vector $\mathrm{X}$ of continuous variables contains some components on which the objective function is differentiable or unconstrained. Then we can place these components on the lower level and use more effective algorithm for minimum point search (for example gradient algorithm or unconstrained one).

The second idea is the combination of both approaches: decomposition and unification. As it was mentioned above, the presence of boolean variables leads to the bad work of discrete algorithm if we use the unification to integer variables. The outcome is to solve two-level problem instead of one-level one, first level containing the boolean variables and second one containing unified discrete and continuous variables.

We plan also to develop the service possibilities of the software system to ensure a support of decision making in choice of suitable way of optimization and appropriate algorithmic tool.

\section{REFERENCES}

Abramovich, K. (1995) Load Planning for Ground-Based Control Complex of Orbital Group of Communication Satellites. Diploma work. Siberian Aerospace Academy, Krasnoyarsk.

Dolezal, J. and Fidler, J. (1992) Dialogue System OPti A for Minimization of Functions of Several Variables. UTIA, Prague.

Semenkin, E. (1992) Search Discrete Optimization for CAD of Spacecrafts, in OptimizationBased Computer-Aided Modelling and Design (eds. A.J.M. Beulens, J. Dolezal and H.-J. Sebastian), IFIP Working Group 7.6, Dagstuhl.

Semenkin, E. and Semenkina, O. (1994) OPti A Software Extension by Discrete Optimization Algorithms, in Optimization-Based Computer-Aided Modelling and Design (eds. J. Dolezal and J. Fidler), IFIP Working Group 7.6, Prague.

Semenkina, O. (1995) Search Techniques for Synthesis of Spacecrafts' Control Systems. Ph.D. thesis. Siberian Aerospace Academy, Krasnoyarsk. (In Russian). 